PROCEEDINGS OF THE

AMERICAN MATHEMATICAL SOCIETY

Volume 127, Number 10, Pages 3091-3094

S 0002-9939(99)04854-6

Article electronically published on April 28, 1999

\title{
A COUNTABLE NONDISCRETE TOPOLOGICAL FIELD WITHOUT NONTRIVIAL CONVERGENT SEQUENCES
}

\author{
ALEXANDER SHIBAKOV
}

(Communicated by Alan Dow)

\begin{abstract}
A construction of a space in the title is presented, answering a question asked by A. Arkhangel'skii and D. Shakhmatov.
\end{abstract}

\section{$\S 1$. INTRODUCTION}

In [S2] D. Shakhmatov attributes the following question to A. Arkhangel'skii:

Question 1.1. Does there exist a nondiscrete countable topological field without nontrivial convergent sequences?

The following argument ${ }^{1}$ shows how to settle a similar question for topological groups. For an arbitrary infinite abelian group $G$, following van Douwen (see [vD]), denote by $G^{\#}$ the group $G$ endowed with the maximal totally bounded topology. The fact that $G^{\#}$ has no nontrivial convergent sequences follows from [vD, Theorem 1.1.3(c)]. The motivation for asking the question above lies in the fact that the topological field structure puts much more restriction on the topological properties of subsets of the field than the topological group structure (see [S2]). Thus it is pointed out in [S2] that in a topological field where no point is $G_{\delta}$ (such as an ultrapower of the real line with the order topology) all convergent sequences are trivial.

In this paper we prove the following theorem which affirmatively answers the question above:

Theorem 1.2. Any infinite countable field admits a nondiscrete topology without nontrivial convergent sequences.

We use standard notation. In what follows $\mathbb{F}$ denotes a countable metrizable nondiscrete commutative field, $\mathcal{C}$ is the family of all compact subsets of $\mathbb{F}$, and $\mathcal{B}$ is a countable base of open sets for the topology of $\mathbb{F}$. Set $\mathcal{B}^{+}=\{\bar{U}: U \in \mathcal{B}, 0 \notin \bar{U}\}$. We will assume that the base $\mathcal{B}$ has the property that $\mathcal{B}^{+}$is closed under taking ${ }^{-1}$, i.e. if $U \in \mathcal{B}^{+}$, then $U^{-1} \in \mathcal{B}^{+}$. If $K_{0}, K_{1} \subseteq \mathbb{F}$, then $K_{0} \cdot K_{1}=\left\{k_{0} \cdot k_{1}: k_{0} \in\right.$ $\left.K_{0}, k_{1} \in K_{1}\right\} \subseteq \mathbb{F}$, the sets $K_{0}+K_{1}$ and $K_{0}^{-1}$ are defined similarly. Note that it follows from the definition above that $\varnothing^{-1}=\varnothing, K+\varnothing=K \cdot \varnothing=\varnothing$ for any

Received by the editors March 25, 1997 and, in revised form, December 12, 1997.

1991 Mathematics Subject Classification. Primary 54H13; Secondary 54A20.

Key words and phrases. Topological field, convergent sequence.

${ }^{1}$ The author is thankful to the referee for suggesting this construction.

(C)1999 American Mathematical Society 
$K \subseteq \mathbb{F}$. We write $S \rightarrow x$ if $S \cup\{x\}$ is homeomorphic to a compact space with a unique nonisolated point $x$.

If $X$ is a topological space and $\mathcal{K}$ is a family of subsets of $X$, then it is said that $\mathcal{K}$ determines the topology of $X$ if every $H \subseteq X$ such that $H \cap K$ is closed for every $K \in \mathcal{K}$ is closed in $X$. We use the following standard facts (see $[\mathrm{M}]$ ):

(1) if the topology of $X$ is determined by a countable family $\mathcal{K}_{X}$ of compact subsets of $X$ and the topology of $Y$ is determined by a countable family $\mathcal{K}_{Y}$ of compact subsets of $Y$, then the topology of $X \times Y$ is determined by the family $\left\{K \times P: K \in \mathcal{K}_{X}, P \in \mathcal{K}_{Y}\right\}$

(2) if the restriction of $p: X \rightarrow Y$ on $K \subseteq X$ is continuous for every $K \in \mathcal{K}$, where $\mathcal{K}$ determines the topology of $X$, then $p$ is continuous.

\section{$\S 2$. ExAMPLE}

Let us define the following operations on arbitrary subsets $K, K_{0}$, and $K_{1}$ of $\mathbb{F}$ :

(3) $\left(K_{0}, K_{1}\right) \mapsto K_{0}+K_{1}$;

(4) $\left(K_{0}, K_{1}\right) \mapsto K_{0} \cdot K_{1}$;

(5) $K \mapsto\{q\}$, where $q \in \mathbb{F}$;

(6) $K \mapsto(K \cap O)^{-1}$, where $O \in \mathcal{B}^{+}$.

Let $\left\{T_{i}\right\}_{i \in \omega}$ list all $T: P(\mathbb{F})^{n_{T}} \rightarrow P(\mathbb{F})$ obtained as compositions of (3)-(6), where $n_{T}$ is the number of arguments of $T$ and $P(\mathbb{F})$ is the power set of $\mathbb{F}$. Abusing this notation we will write $T_{i}(K)$ instead of $T_{i}(K, \ldots, K)$ meaning that all the arguments of $T_{i}$ are the same. It is obvious that $T_{i}$ 's preserve both $\mathcal{C}$ and $[\mathbb{F}]^{<\omega}$. Now let $K$ be a compact subset of $\mathbb{F}$. Denote

$$
C(K)=\left\{T_{i}(K): i \in \omega\right\} .
$$

Then $C(K)$ consists of compact subsets of $\mathbb{F}$, contains all singletons, and is closed under (3)-(6). Let us introduce a new topology on $\mathbb{F}$ as follows: $H \subseteq \mathbb{F}$ is closed if $H \cap C$ is closed for each $C \in C(K)$. Then in the new topology each member of $C(K)$ has the original topology induced by $\mathbb{F}$. Denote $\mathbb{F}$ with this topology as $F(K)$. Now $C(K)$ determines the topology of $F(K)$.

The idea of the short proof of the following lemma presented here is borrowed from $[\mathrm{MMO}]$ :

Lemma 2.1. The topology of $F(K)$ is the maximal field topology on $\mathbb{F}$ inducing the original topology on $K$.

Proof. To show that $F(K)$ is a topological field it is enough to prove that the mappings $p_{1}: F(K) \times(F(K) \backslash\{0\}) \rightarrow F(K)$ and $p_{2}: F(K) \times F(K) \rightarrow F(K)$, where $p_{1}\left(x_{1}, x_{2}\right)=x_{1} \cdot x_{2}^{-1}$ and $p_{2}\left(x_{1}, x_{2}\right)=x_{1}-x_{2}$ are continuous. Put

$$
C^{+}(K)=\left\{T_{i}(K) \cap O: i \in \omega, O \in \mathcal{B}^{+}\right\} .
$$

Then, since $\mathcal{B}^{+}$is closed under taking ${ }^{-1}, C^{+}(K) \subseteq C(K)$ and it is immediate that $C^{+}(K)$ determines the topology of $F(K) \backslash\{0\}$. Now both $C(K)$ and $C^{+}(K)$ are countable families of compact subsets of $\mathbb{F}$ so it follows from (1) and (2) that to prove the continuity of $p_{1}$ it is enough to show that the restriction of $p_{1}$ on $T_{i}(K) \times\left(T_{j}(K) \cap O\right)$ is continuous for every $i, j \in \omega, O \in \mathcal{B}^{+}$. Now

$$
p_{1}\left(T_{i}(K) \times\left(T_{j}(K) \cap O\right)\right)=T_{i}(K) \cdot\left(T_{j}(K) \cap O\right)^{-1} \in C(K)
$$

(since $\left(T_{j}(K) \cap O\right)^{-1} \in C(K)$ as a result of application of one of the operations (6) to a member of $C(K))$. Thus both $T_{i}(K) \times\left(T_{j}(K) \cap O\right)$ and $p_{1}\left(T_{i}(K) \times\left(T_{j}(K) \cap O\right)\right)$ 
have the topology induced by $\mathbb{F}$. Since $p_{1}$ is continuous in the original topology of $\mathbb{F}$, the argument for $p_{1}$ is complete.

Similarly, for $p_{2}$ one observes that $p_{2}\left(T_{i}(K) \times T_{j}(K)\right)=T_{i}(K)-T_{j}(K)=T_{i}(K)+$ $\{-1\} \cdot T_{j}(K) \in C(K)$ since $\{-1\} \in C(K)$ by $(5)$.

Now if $\tau$ is a field topology on $\mathbb{F}$ inducing the original topology on $K$, then for every $i \in \omega$ the set $T_{i}(K)$ is compact. So if $H \subseteq \mathbb{F}$ is closed in $\tau$, then for every $i \in \omega$ the set $H \cap T_{i}(K)$ is closed in the usual topology of $\mathbb{F}$. Thus $H$ is closed in the topology of $F(K)$.

Lemma 2.2. For any $i \in \omega$ the operation $T_{i}$ satisfies the following properties (below $n$ stands for the number of arguments of $\left.T_{i}\right)$ :

(7) for any finite $f_{1} \subseteq \mathbb{F}, \ldots, f_{n} \subseteq \mathbb{F}$ the set $T_{i}\left(f_{1}, \ldots, f_{n}\right)$ is finite;

(8) for any $q \in T_{i}\left(K_{1}, \ldots, K_{n}\right)$ there are finite $f_{1} \subseteq K_{1}, \ldots, f_{n} \subseteq K_{n}$ such that $q \in T_{i}\left(f_{1}, \ldots, f_{n}\right)$;

(9) if $K_{1}^{\prime} \subseteq K_{1}, \ldots, K_{n}^{\prime} \subseteq K_{n}$, then $T_{i}\left(K_{1}^{\prime}, \ldots, K_{n}^{\prime}\right) \subseteq T_{i}\left(K_{1}, \ldots, K_{n}\right)$;

(10) for any open $U \subseteq \mathbb{F}$ such that $T_{i}\left(K_{1}, \ldots, K_{n}\right) \subseteq \bar{U}$ there are open $V_{1}, \ldots, V_{n}$ such that $K_{1} \subseteq V_{1}, \ldots, K_{n} \subseteq V_{n}$ and $T\left(V_{1}, \ldots, V_{n}\right) \subseteq U$.

Proof. It is easy to show that taking compositions preserves properties (7)-(10). So to prove the lemma it is enough to show that operations (3)-(6) have properties (7)-(10). The only nontrivial case is (10). For operations (3) and (4) the property immediately follows from the well known property of multiplication (resp. addition): whenever $K_{0} \cdot K_{1} \subseteq U$ (resp. $K_{0}+K_{1} \subseteq U$ ) for compact $K_{0}, K_{1}$, and an open $U$, then there exist open sets $V_{i} \supseteq K_{i}$ such that $V_{0} \cdot V_{1} \subseteq U$ (resp. $\left.V_{0} \cdot V_{1} \subseteq U\right)$. The case of (5) is trivial. To show (10) for operations (6) suppose that $(P \cap O)^{-1} \subseteq U$ for some $O \in \mathcal{B}^{+}$, compact $P$, and open $U$. Since $0 \notin O^{-1}$, we may assume without loss of generality that $0 \notin U$. Put $V=\mathbb{F} \backslash\left(O \backslash U^{-1}\right)$. Obviously $V$ is an open subset of $\mathbb{F}$ and a routine check shows that $(V \cap O)^{-1} \subseteq U$ and $P \subseteq V$.

Lemma 2.3. Let $S \rightarrow 0$ in the usual topology of $\mathbb{F}$. Then there are infinite $K \subseteq S$ and $D \subseteq S$ such that $D$ is a closed discrete subset of $G(K \cup\{0\})$.

Proof. Let us construct for every $i \in \omega$ points $x_{i}, z_{i} \in S$ such that

(11) $x_{i} \neq x_{j}$ and $z_{i} \neq z_{j}$ if $i \neq j$;

(12) if $n \geq k$ and $l \geq k$, then $z_{l} \notin T_{k}\left(\bigcup_{j \leq n}\left\{x_{j}\right\} \cup\{0\}\right)$.

Let $x_{0}$ be an arbitrary point of $\mathbb{F}$ and choose $z_{0}$ so that $z_{0} \notin T_{0}\left(\left\{x_{0}\right\} \cup\{0\}\right)$. Suppose $x_{i}, z_{i}$ have been constructed for $i<n$ so that they satisfy (11)-(12). It follows from (7) that the set $\bigcup_{i \leq n} T_{i}\left(\bigcup_{j<n}\left\{x_{j}\right\} \cup\{0\}\right)$ is finite. So there exists $z_{n} \in S$ such that

$$
z_{n} \notin \bigcup_{i \leq n} T_{i}\left(\bigcup_{j<n}\left\{x_{j}\right\} \cup\{0\}\right) \cup\left\{z_{i}: i<n\right\} .
$$

Now by (10) for every $l, k \in \omega$ such that $n \geq l \geq k$ there exists an open $U_{l, k} \ni 0$ such that

$$
z_{l} \notin T_{k}\left(\bigcup_{j<n}\left\{x_{j}\right\} \cup\left(U_{l, k} \cap S\right) \cup\{0\}\right) .
$$

Put $U=\bigcap_{n>l>k} U_{l, k}$. Now if $x_{n} \in(S \cap U) \backslash\left\{x_{i}: i<n\right\}$, then (11)-(12) are easy to check. Put $K=\left\{x_{i}: i \in \omega\right\}$. Then $K \rightarrow 0$ and (12) and imply that for any $i \in \omega$ and $j \in \omega$ such that $j>i$

$$
z_{j} \notin T_{i}(K \cup\{0\}) .
$$


So $D=\left\{z_{i}: i \in \omega\right\}$ intersects each element of $C(K \cup\{0\})$ in a finite subset and therefore is a closed discrete subset of $F(K \cup\{0\})$.

Proof of Theorem 1.2. Let $\mathbb{F}$ be an infinite countable field. By [W, Theorem 6, p. 23 ] there exists a nondiscrete topology on $\mathbb{F}$ which can be made metrizable using the main result of [S1]. Using Zorn's lemma, construct a maximal nondiscrete field topology (i.e. a field topology such that any finer field topology is discrete) $\tau$ on $\mathbb{F}$ which is finer than the metrizable one.

Suppose $S \rightarrow 0$ in $\tau$ for some infinite $S \subseteq \mathbb{F}$. By Lemma $2.1 \tau$ is the topology of $F(S \cup\{0\})$. Lemma 2.3 implies that there is $K \subseteq S$ such that the topology of $F(K \cup\{0\})$ is finer than the topology of $F(S \cup\{0\})$, a contradiction.

The author is thankful to Professor G. Gruenhage for his valuable remarks and suggestions, to Professor D. Shakhmatov for helpful communication about the problem, and to the referee for suggesting various improvements to the proof.

\section{REFERENCES}

[vD] E. van Douwen, The maximal totally bounded topology on $G$ and the biggest minimal G-space for Abelian groups $G$, Topology Appl. 34 (1990), 69-91.

[M] E. Michael, Bi-quotient maps and Cartesian products of quotient maps, Ann. Inst. Fourier (Grenoble) 18 (1968), 287-302. MR 39:6277

[MMO] J. Mack, S. Morris, E. Ordman, Free topological groups and the projective dimension of a locally compact abelian group, Proc. Amer. Math. Soc. 40 (1973), 303-308. MR 47:8755

[S1] D. B. Shakhmatov, Condensations of universal topological algebras preserving continuity of operations and decreasing weights, Moscow Univ. Math. Bull. 39 (1984), 42-45. MR 85h:22003

[S2] D. B. Shakhmatov, The structure of topological fields and cardinal invariants, Trans. Moscow Math. Society 50 (1987), 249-259. MR 89c:54076

[W] W. Wiesław, Topological fields, Marcel Dekker, Inc., New York, 1988. MR 89i:12007

Department of Mathematics, Box 5054, Tennessee Technological University, CoOkeville, Tennessee 38505

E-mail address: ashibakov@tntech.edu 